$$
\begin{gathered}
\text { CONF }=960808-2 \\
\text { ANL/MSD/CP }--91231 \\
\text { Onset of Bulk Pinning in BSCCO Single Crystals }
\end{gathered}
$$

\author{
C.J. van der Beek, ${ }^{*}$ M.V. Indenbom, V. Berseth, T.W. Li, $\underset{\ddagger}{\ddagger}$ and W. Benoit \\ IGA, Ecole Polytechnique Fédérale de Lausanne, CH-1015 Lausanne, Switzerland \\ ${ }^{*}$ Materials Science Division and Science and Technology Center for Superconductivity \\ Argonne National Laboratory, Argonne, Illinois 60439 \\ $\ddagger$ Kamerlingh Onnes Laboratory, Leiden University, Leiden, The Netherlands
}

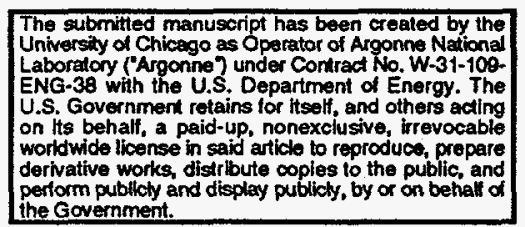

Conference on Materials Aspects of Organic and High Temperature Superconductors (MOS '96), Karlsruhe, Germany, August 2-6, 1996, to appear in Journal of Low Temperature Physics

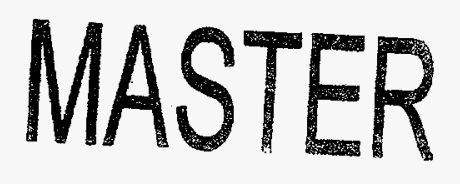

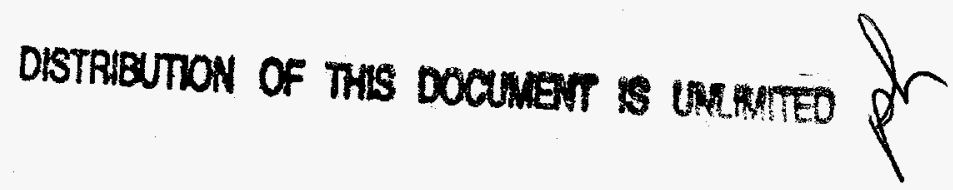

\footnotetext{
Work supported by the NSF-Office of Science and Technology Centers for Superconductivity under contract \#DMR91-20000, by the U.S. Department of Energy, Office of Basic Energy Sciences-Materials Sciences under contracts \#W-31-109-ENG-38 (CJvdB), and by the Swiss National Foundation under Grant No. 21-42015.94.
} 


\section{DISCLAIMER}

This report was prepared as an account of work sponsored by an agency of the United States Government. Neither the United States Government nor any agency thereof, nor any of their employees, makes any warranty, express or implied, or assumes any legal liability or responsibility for the accuracy, completeness, or usefulness of any information, apparatus, product, or process disclosed, or represents that its use would not infringe privately owned rights. Reference herein to any specific commercial product, process, or service by trade name, trademark, manufacturer, or otherwise does not necessarily constitute or imply its endorsement, recommendation, or favoring by the United States Government or any agency thereof. The views and opinions of authors expressed herein do not necessarily state or reflect those of the United States Government or any agency thereof. 


\section{DISCLAIMER}

Portions of this document may be illegible in electronic image products. Images are produced from the best available original document. 


\title{
Onset of Bulk Pinning in BSCCO Single Crystals
}

\author{
C.J. van der Beek, ${ }^{*}$ M.V. Indenbom, V. Berseth, T.W. Li, \\ and W. Benoit \\ IGA, Ecole Polytechnique Fédérale de Lausanne, CH-1015 Lausanne, Switzerland \\ -Materials Science Division and Science and Technology Center for \\ Superconductivity, Argonne National Laboratory, Argonne, IL 60439, U.S.A. \\ † Kamerlingh Onnes Laboratory, Leiden University, Leiden, the Netherlands
}

The long growth defects often found in $\mathrm{Bi}_{2} \mathrm{Sr}_{2} \mathrm{CaC}_{2} \mathrm{O}_{8}$ "single" crystals effectively weaken the geometrical barrier and lower the field of first flux penetration. This means that the intrinsic (bulk) magnetic properties can be more easily accessed using magnetic measurements. Thus, the onset of strong bulk flux pinning in the sample bulk is determined to lie at $T \approx 40$ $K$, 'indepedent of whether the field strength is above or below the field of the second peak in the magnetisation. (PACS numbers: $74.60 \mathrm{Ge}$ )

\section{INTRODUCTION}

It is now well established that at sufficiently high temperature, magnetic irreversibility in $\mathrm{Bi}_{2} \mathrm{Sr}_{2} \mathrm{CaCu}_{2} \mathrm{O}_{8}$ (BSCCO) single crystals is dominated by 2 geometrical barrier acting against penetration of vortices into the sample. ${ }^{1-3}$ Amongst other properties, the barrier determines the crystals' magnetic acsusceptibility as measured in a modest DC background field. 4,5 As was shown from the analysis of the third harmonic susceptibility $\chi_{3},{ }^{5}$, the susceptibility. resembles that which would be measured for a (super-) conducting ring, a feature that leads to an apparent "negative transmittivity" if the AC flux density is measured locally. ${ }^{4}$ The shielding of the sample interior by the superconducting edge current obscures the behaviour of the vortices in the bulk, and methods to circumvent the effects of the barrier should be considered when one wishes to study vortex pinning in BSCCO at small fields.

The ascending field branch of the irreversible magnetisation originating from an edge- or surface barrier has been shown to depend on the magnetic 

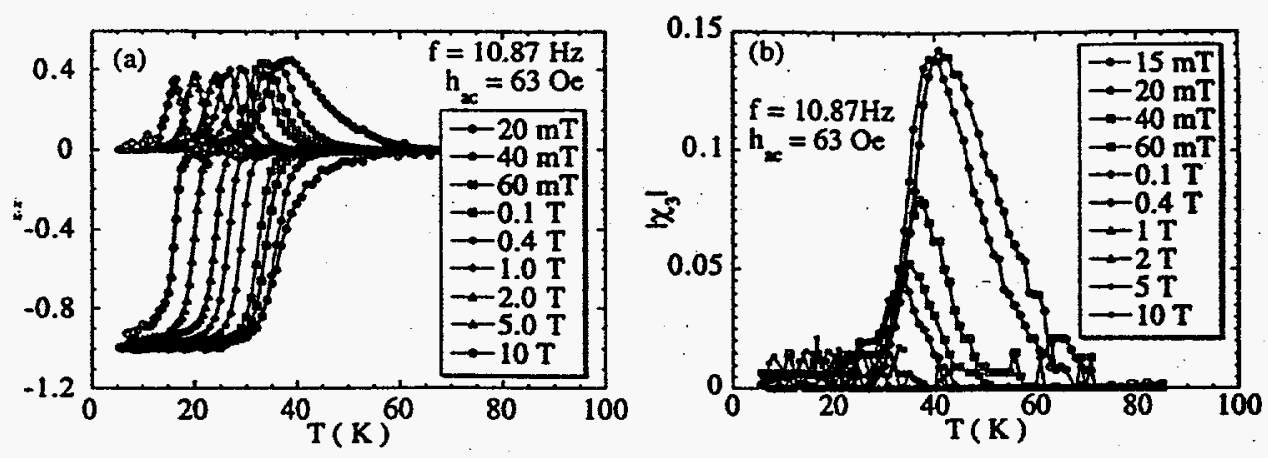

Fig. 1. First (a) and third (b) harmonic AC susceptibility of the BSCCO sample, measured using an AC field of $63 \mathrm{Oe} \mathrm{RMS}$, and $f=10.87 \mathrm{~Hz}$, in DC fields $H_{0}$ between 0.02 and $10 \mathrm{~T}$.

field $H$ as $M \propto H_{-p}^{2} / H$ independently of the origin of the barrier. ${ }^{5} \mathrm{By}$ consequence, the $A C$ susceptibility depends, to a very good approximation, on the $\mathrm{DC}$ and $\mathrm{AC}$ field strengths $H_{0}$ and $h_{a c}$, and on the penetration field $H_{p}$ in the combination $H_{0} h_{a c} / H_{p}^{2}$ only. The ac screening resulting from the barrier can thus be similarly suppressed by lowering $H_{p}$ or by increasing $h_{a c}$.

A method to reduce $H_{p}$ in the case of the geometrical barrier is to shape the single crystalline sample into a prism. ${ }^{6,7}$ However, $H_{p}$ can also be reduced - because of naturally occuring crystalline defects. For example, low angle grain boundaries often present in samples grown by the travelling solvent floating zone technique act as ducts for vortices and facilitate flux entry into the sample..$^{8,9}$ Due to the opposite polarity of the screening currents on either side of the grain boundary, vortices are bent only slightly when they enter the bulk. The condition that $H=H_{c 1}$ over the whole sample thickness ${ }^{2}$ is quickly satisfied as $H_{0}$ is increased, therefore $H_{p}$ is low.

In this contribution, we present results on an as-grown BSCCO crystal with a low $H_{p}$. The screening due to the edge-shape barrier is suppressed by applying a large AC field, until a lower temperature limit is reached. We interpret this limit as the onset of observable flux pinning in the bulk of the crystal.

\section{EXPERIMENTAL DETAILS}

The sample used, the same as in Ref. [5], was grown using the travellingsolvent floating zone technique. Its dimensions are $2.71(1) \times 1.84(w) \times$ $0.081(\mathrm{t}) \mathrm{mm}^{3}$, and $T_{c} \approx 86.5 \mathrm{~K}$. A magneto-optical investigation revealed the presence of some enlongated defects in the interior, similar to the ones reported in Ref. [7]. These defects, a result of the particular growth process, ${ }^{9}$ 
Onset of Bulk Pinning in BSCCO

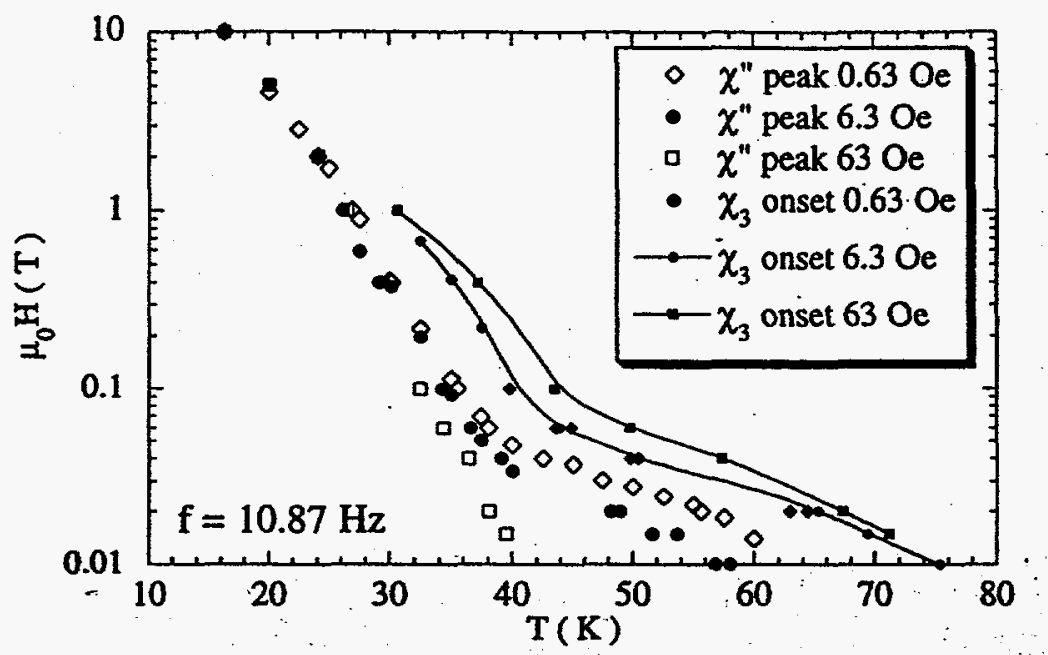

Fig. 2. The positions in the $(H, T)$-plane of the peaks in $\chi^{\prime \prime}$ and $\left|\chi_{3}\right|$ (which very nearly coincide) measured using an $\mathrm{AC}$ field of frequency $f=10.87$. Hz and $A C$ amplitudes of $0.63,6.3$, and $63 \mathrm{Oe}$. The lines show the position of the irreversibility line tracked by the third harmonic onset.

are not visible from the surface, and provide preferential paths for entering flux at temperatures in excess of $30 \mathrm{~K}$ and fields above $50 \mathrm{Oe}$. AC susceptibility measurements, using frequencies $1.36<f<5565 \mathrm{~Hz}$, and AC field amplitudes $0.63<h_{a c}<63 \mathrm{Oe}$, were performed in DC fields $H_{0}$ of up to $10 \mathrm{~T}$ using the AC susceptometer at Argonne. The AC field was always oriented colinearly with the DC field, parallel to the sample c-axis.

\section{RESULTS AND DISCUSSION}

Figure 1 shows the AC susceptibility of the BSCCO sample as measured with $f=10.87 \mathrm{~Hz}$ and $h_{a c}=63 \mathrm{Oe}$. The susceptibility curves are sharp and nearly parallel for $\mu_{0} H_{0}>40 \mathrm{mT}$, and the low-temperature flanks of the $\left|\chi_{3}\right|$-peaks nearly coincide for all $\mathrm{DC}$ fields. This is in contrast to earlier results at much lower AC amplitudes, where the low-field susceptibility curves are much broader and depend strongly on $H_{0} .5,10$ Whereas at low $h_{a c}$, an increase of $\mu_{0} \mathrm{H}_{0}$ from 10 to $100 \mathrm{mT}$ causes the screening transition and the $\chi^{\prime \prime}$ and $\left|\chi_{3}\right|$-peaks to shift from 75 to $40 \mathrm{~K},{ }^{11}$ the present results show a near field-independence in the same field range. The only remnant of the field dependence is observed in the high temperature tails and, equivalently, in the onset of $\left|\chi_{3}\right|$.

Figure 2 shows the positions of the peaks in $\chi^{\prime \prime}$ and $\left|\chi_{3}\right|$, and those of the $\chi_{3}$ onset, in the $(H, T)$-plane for $f=10.87 \mathrm{~Hz}$ and different $\mathrm{AC}$ amplitudes. 

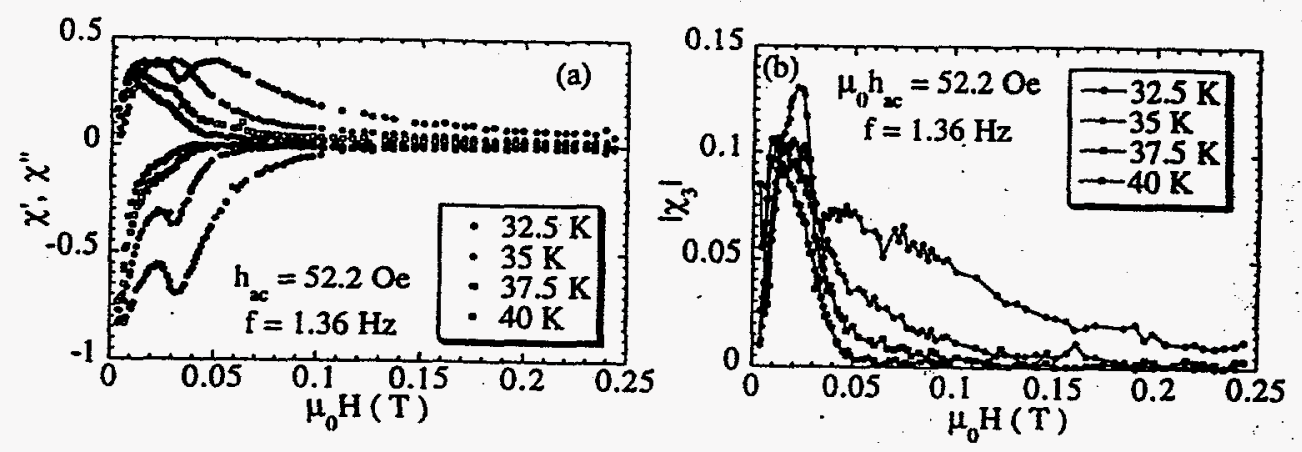

Fig. 3. First (a) and third (b) harmonic AC susceptibility of the BSCCO sample, measured using an AC field of $52.2 \mathrm{Oe} \mathrm{RMS}$, and $f=1.36 \mathrm{~Hz}$, at temperatures between 32.5 and $40 \mathrm{~K}$.

As $h_{a c}$ is increased, the locus of peak fields, $H_{\text {peak }}(T)$, drastically changes its shape: at low and intermediate AC amplitudes, it shows a crossover from a very rapid drop to the $\left(1-T / T_{c}\right)^{2}$-behaviour characteristic for the barrier screening. At the highest amplitude only the steep drop remains, without any particular features. The magnetic "irreversibility line" tracked as the onset of $\left|\chi_{3}\right|$ moves to higher temperature as $h_{a c}$ is increased.

Since in all measurements, $h_{a c}$ is appreciably smaller than $H_{0}$, the sup$\therefore$ pression of the low field part of $H_{\text {peak }}(T)$-line cannot be explained merely as a result of the increased total field $H_{0}+h_{a c}$. Rather, much of the screening associated with the barrier is suppressed when a sufficiently large $\mathrm{AC}$ field (of the order $50 \mathrm{Oe}$ ) is applied: only the susceptibility "tails" located between the peak temperature and the third harmonic onset remain. Appreciable screening of the AC field occurs only when $h_{a c}$ becomes of the order $j_{p} d$, where $j_{p}$ is the bulk current due to pinning. and $d$ is the sample thickness. The locus of $\chi^{\prime \prime}$-peaks measured with $h_{a c}=63$ Oe corresponds to a line of constant current density, $j \approx 6.2 \times 10^{7} \mathrm{Am}^{-2}$. More specifically, it corresponds to the line where the shielding current density $j_{p} \sim 6 \times 10^{7} \mathrm{Am}^{-2}$ due to pinning is greater or equal to the barrier current at all $\mathrm{DC}$ field strengths. We can thus estimate the penetration field for our sample, ${ }^{3} H_{p} \approx \frac{1}{2} j d \sqrt{d / W}<15$ Oe ( $W$ is the mean sample width), 3 times lower than in other crystals. ${ }^{1}$

Pinning is seen to emerge at temperatures between 35 and $40 \mathrm{~K}$, irrespective of the magnetic field. This is quite surprising, because BSCCO shows quite different pinning characteristics depending on whether the field is above or below a certain threshold value. Fig. 3(a) shows that between 30 and $40 \mathrm{~K}, \chi^{\prime}$ has a clear "second maximum" (at $\mu_{0} H_{s p} \approx 35 \mathrm{mT}$ ), and $\chi^{\prime \prime}$ has a two-peak structure. These features correspond to a maximum in the 
Onset of Bulk Pinning in BSCCO

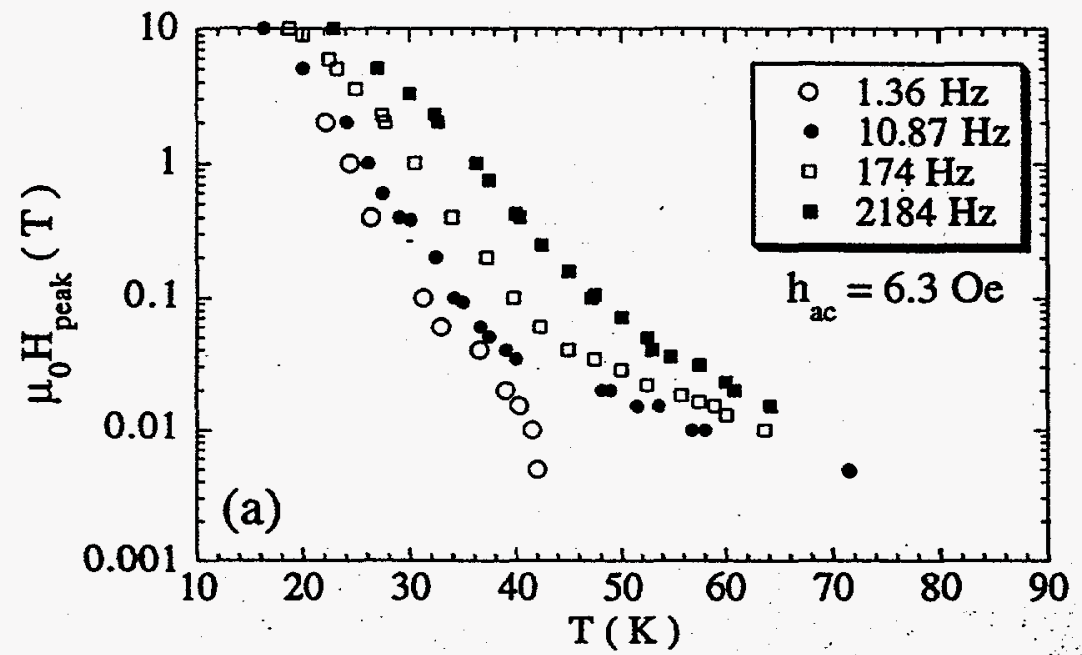

Fig. 4. Position in the $(H, T)$-plane of the peak in $\chi^{\prime \prime}$ measured with $h_{a c}=6.3 \mathrm{Oe}$, and frequencies between $1.36 \mathrm{~Hz}$ and $2184 \mathrm{~Hz}$. These data indicate the apparently lower screening efficiency of the edge- shape barrier at lower $\mathrm{AC}$ frequencies.

bulk shielding current, and to the "second peak". that is usually observed in the DC magnetisation of BSCCO single crystals. ${ }^{1}$ At $H_{s p}$, the third harmonic amplitude shows a sudden drop, indicating that at fields above $\boldsymbol{H}_{\boldsymbol{s}}$ the sample's $I(V)$-curve is much more linear than at fields below it, an effect that is partly due to the sudden vanishing of the geometrical barrier at the fundamental vortex lattice phase transition. ${ }^{12-14}$

The screening due to the geometrical barrier also apparently changes by changing the $\mathrm{AC}$ frequency, as shown in Fig. 4. This effect is unexpected since penetration over the barrier in principle requires vortex motion over a macroscopically large distance, which in our AC experiment, carried out well above the field of first flux penetration, should be comparable to the sample thickness $d \approx 81 \mu \mathrm{m}$. The associated activation barrier was assumed to be prohibitively large. ${ }^{3}$ Two hypotheses can be advanced in order to explain the observed frequency dependence. First, flux perretration into the sample could be determined by a few "weak" points on the edge or along the defects in the interior, sufficiently weak to permit vortex entry through thermal activation, resulting in a frequency-dependent penetration field. Second, it is known from magneto-optical observations that pinning remains present above 40 $\mathrm{K},{ }^{14}$ and that this opposes the internal redistribution of flux during the $\mathrm{AC}$ cycle. Thermal activation from the pinning potential is then responsible for the observed frequency dependence, the curves measured at lower frequency corresponding more closely to what is to be expected for screening by the 


\section{C.J. van der Beek et al.}

geometrical barrier only. In crystals with a low penetration field, such as the one under study, pinning should dominate over barrier irreversibility more rapidly when frequency is increased than in crystals with a high $H_{p}$.

In summary, the magnetic screening due to the geometrical barrier can be suppressed by the application of a sufficiently large AC field. This procedure allows the observation of the onset of strong bulk flux pinning inBSCCO crystals. Pinning was found to appear at the same temperature for fields above and below the second peak in the magnetisation.

\section{ACKNOWLEDGMENTS}

This work was supported by the National Science Foundation through the Science and Technology Center for Superconductivity (Cooperative Agreement No. DMR91-20000) (C.J. v.d.B.), and by the Swiss National foundation under Grant No. 21-42015.94.

\section{REFERENCES}

1. N. Chikumoto, M. Konczykowski, N: Motohira, and A.P. Malozemoff, Phys. Rev. Lett. 69, 1260 (1992).

2. M.V. Indenbom, G. D'Anna, M.-O. André, W. Benoit, H. Kronmüller, T.W. Li, and P.H. Kes, in Proceedings of the $7^{\text {th }}$ Int. Workshop on Critical Currents in Superconductors, Alpbach, Austria, Jan. 24-27, 1994, Edited by H.W. Weber

- (World Scientific, 1994), pp. 327-330.

3. E. Zeldov, A.I. Larkin, V.B. Geshkenbein, M.Konczykowski, D. Majer, B. Khaykovich, V.M. Vinokur, and H.Strikhman, Phys. Rev. Lett. 73, 1428 (1994).

4. N. Morozov, E. Zeldov, D. Majer, and B. Khaykovich, Phys. Rev. Lett. 76, 138 (1996).

5. C.J. van der Beek, M.V. Indenbom, G.D'Anna, and W. Benoit, Physica C 258, 105 (1996).

6. W. DeSorbo and W.A. Healy, Cryogenics 4, 257 (1964).

7. D. Majer, E. Zeldov, and M. Konczykowski, Phys. Rev. Lett. 75, 1166 (1995).

8. M.R. Koblischka, R.J. Wijngaarden, D.G. de Groot, R. Griessen, A.A. Menovsky, and T.W. Li, Physica C 249, 339 (1995).

9. M.V. Indenbom, C.J. van der Beek, V. Berseth, Th. Wolf, H. Berger, T.W. Li, and $W$. Benoit, this conference.

10. J. van den Berg, C.J. van der Beek, P.H. Kes, J.A. Mydosh, M.J.V. Menken, and A.A. Menovsky, Superc. Sci. Techn. 1, 249 (1989).

11. C.J. van der Beek and P.H. Kes, Phys. Rev. B 43, 13032 (1991).

12. M.V. Indenbom, Physica C 235-240, 201 (1994).

13. E. Zeldov, D.Majer, M. Konczykowski, V.B. Geshkenbein, V.M. Vinokur, and H. Strikhman, Nature 375, 373 (1995).

14. M.V. Indenbom, C.J. van der Beek, V. Berseth, M. Konczykowski, N. Motohira, H. Berger, and W. Benoit, this conference. 